ज्ञ FRANÇAISE

$\supset \mathrm{DE}$

때 PÉDAGOGIE

\section{Revue française de pédagogie}

Recherches en éducation

178 | janvier-mars 2012

Les politiques de lutte contre les inégalités scolaires d'un pays à l'autre

\title{
Dictée notée ou dictée non notée ? Analyse des erreurs orthographiques des élèves en situation scolaire
}

Marked dictation or not? Analysis of the spelling mistakes of schooled pupils

¿Dictado evaluado o dictado no evaluado con una nota? Análisis de los errores de ortografía de los alumnos en situación escolar

Diktat mit oder ohne Note? Analyse der Rechtschreibfehler der Schüler an der

Schule

Marie-Christine Toczek, Michel Fayol et Marion Dutrévis

\section{(2) OpenEdition}

Journals

Édition électronique

URL : http://journals.openedition.org/rfp/3560

DOI : $10.4000 / \mathrm{rfp} .3560$

ISSN : 2105-2913

Éditeur

ENS Éditions

Édition imprimée

Date de publication : 15 mars 2012

Pagination : 85-96

ISBN : 978-2-84788-372-5

ISSN : 0556-7807

Distribution électronique Cairn

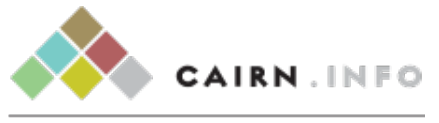

CHERCHER, REPÉRER, AVANCER.

\section{Référence électronique}

Marie-Christine Toczek, Michel Fayol et Marion Dutrévis, « Dictée notée ou dictée non notée ? Analyse des erreurs orthographiques des élèves en situation scolaire », Revue française de pédagogie [En ligne], 178 | janvier-mars 2012, mis en ligne le 15 mars 2015, consulté le 06 mai 2019. URL : http:// journals.openedition.org/rfp/3560; DOI : 10.4000/rfp.3560 


\title{
Dictée notée ou dictée non notée ? Analyse des erreurs orthographiques des élèves en situation scolaire
}

\author{
Marie-Christine Toczek, Michel Fayol \\ et Marion Dutrévis
}

L'exercice de dictée permet-il une bonne mise en œuvre des compétences orthographiques des élèves ? Telle est la question centrale de cette recherche. Plus précisément, nous avons évalué les effets des trois situations scolaires au cours desquelles des élèves ont été conduits à écrire un texte sous dictée : un contexte "orthographe-dictée » où l'on testera d'abord l'effet de l'annonce d'une dictée notée, et ensuite l'annonce d'une dictée non notée ; enfin un contexte non lié à l'orthographe. Pour fonder nos hypothèses de travail, nous avons ancré notre recherche sur un ensemble de travaux liés à la régulation sociale des performances scolaires. Or, contrairement à nos attentes, l'analyse des résultats révèle que les situations dont l'enjeu évaluatif est élevé conduisent les élèves à réaliser de meilleures performances orthographiques, et ce quel que soit leur niveau. Aussi une discussion tentera-t-elle de construire de nouvelles hypothèses explicatives. Les résultats obtenus semblent révéler qu'un des problèmes majeurs de l'orthographe n'est peut-être pas d'avoir des compétences mais d'être en mesure de les mettre en œuvre en situation.

Mots-clés (TESE) : orthographe, compétences d'écriture, évaluation sommative, notation, milieu d'apprentissage.

Depuis sa généralisation à l'école dans la deuxième moitié du XIX siècle par le biais du certificat d'études (Chervel, 2006), la dictée représente l'exercice scolaire le plus célèbre, un exercice transgénérationnel. C'est véritablement un symbole brandi par différents ministres (pour juger de la qualité de l'école), par les éditeurs, les publicitaires ou certains artistes. La dictée fait figure de tradition indéracinable dans notre système scolaire. Cabanel (2002) explique comment cet exercice est devenu emblématique au cours de I'histoire de l'éducation, avec le zéro éliminatoire qui a longtemps été le principal obstacle à l'obtention du certificat d'études. Aujourd'hui encore, la dictée semble représenter une épreuve face à laquelle les élèves doivent fournir des efforts et gérer la peur des fautes, la peur de l'échec (Manesse, 2007). Mais quelle est la place de la dictée aujourd'hui dans les pratiques scolaires ? Manesse tente de répondre à cette question. En effet, même si nous sommes dans l'incapacité de rendre compte de manière quantitative des pratiques des enseignants sur cette question, il semble que la dictée soit toujours « vivante » dans les classes. Selon cette chercheuse, la dictée fait bien partie des pratiques courantes dans les classes aujourd'hui et elle doit sa longévité à deux types d'arguments. Le premier est lié à la transmission du patrimoine litté- 
raire. La dictée représente un objet de langue, un extrait d'une œuvre littéraire, un texte. Et de ce fait, elle est un véritable vecteur d'acculturation populaire. Le second argument concerne l'aspect ritualisé de l'exercice. Les dictées se déroulent au cours d'une activité collective orchestrée par l'enseignant qui lit ou énonce à haute voix un texte selon un rythme qui permet aux élèves de le copier par écrit. Ce moment correspond à un rituel dont les règles et les critères sont connus de tous les élèves. Selon Manesse, la dictée est le seul exercice ayant résisté sous une forme stable et ritualisée, dans l'école, depuis un siècle et demi. La simplicité de sa mise en œuvre a d'ailleurs permis de le reproduire en famille et dans les médias.

Que disent les programmes actuels à son propos ? Dans les instructions officielles, cet exercice apparaît systématiquement lorsqu'il s'agit d'évaluer les compétences liées à la maîtrise de la langue. Ainsi, dans la compétence 1 du socle commun de connaissances et de compétences (2005) et dans le livret personnel permettant l'évaluation de ces compétences, il est demandé à chaque enseignant de renseigner l'item « écrire lisiblement un texte, sous la dictée ou spontanément, en respectant l'orthographe et la grammaire ". De plus, il est important de noter que toutes les évaluations institutionnelles créées par la direction de l'évaluation, de la prospective et de la performance (DEPP), ainsi que le brevet des collèges, comportent une épreuve de dictée. En somme, cet exercice reste aujourd'hui l'exercice de référence pour évaluer les compétences orthographiques des élèves. Il est aussi bien utilisé au service d'objectifs liés aux évaluations formatives que sommatives. Or, selon les observations réalisées en contexte et selon l'analyse de Manesse, du point de vue des élèves, cet exercice génère soit un sentiment de sécurité chez ceux qui se réjouissent à l'annonce de la dictée, soit, chez d'autres élèves, des émotions négatives liées à l'inquiétude ou la peur d'échouer. Cette auteure affirme d'ailleurs, au regard de ses observations, que les élèves inquiets semblent rassurés lorsqu'ils savent que la dictée ne sera pas notée. Mais au-delà des émotions ressenties, qu'en est-il des effets réels sur la mise en œuvre des compétences orthographiques des élèves ? L'exercice de dictée permet-il une bonne mise en œuvre des compétences orthographiques ? Et lorsqu'il est noté, améliore-t-il ou réduit-il cette mise en œuvre ? À notre connaissance, aucune étude n'a questionné cette pratique pédagogique en contexte scolaire réel afin d'en mesurer les effets réels sur les performances orthographiques des élèves. C'est précisément l'objet de cette recherche.
La tradition scolaire française veut que les erreurs d'orthographe soient le plus souvent comptabilisées, parfois avec des pondérations : une «faute » entière pour les accords, une « demi-faute » pour les erreurs lexicales, et ainsi de suite (Fayol \& Jaffré, 2008). En fait, les critères de la notation de la dictée ont été l'objet de débats multiples, qui seraient hors de propos ici, mais qui soulignent une nouvelle fois l'importance de l'exercice, surtout lorsqu'il est noté. Aucune recherche ne s'étant intéressée à l'activité cognitive mobilisée lors de cet exercice, nous ignorons quels sont les effets des contextes évaluatifs attachés à la dictée. Néanmoins, d'une manière générale, certaines recherches en psychologie sociale de l'éducation, et plus particulièrement dans le domaine de l'influence des contextes évaluatifs, sont susceptibles de nous aider à fonder des hypothèses de travail. En effet, de nombreux travaux attestent de l'influence du contexte sur les performances intellectuelles (par exemple Brunot, Huguet \& Monteil, 2000 ; Gimmig, Huguet, Caverni et al., 2006 ; Monteil \& Huguet, 2002 ; Schmader \& Johns, 2003 ; Steele, 1997). L'ensemble de ces travaux suggère que la mise en œuvre des compétences des élèves n'est pas seulement affectée par leurs aptitudes mais aussi, et de façon majeure, par les contextes sociaux dans lesquels sont recueillies les performances. Ainsi, dès 1991, Monteil et Huguet (voir également Huguet, Brunot \& Monteil, 2001) ont montré que le contexte de présentation d'une tâche était susceptible d'influer différemment sur la performance des élèves en situation de réussite ou d'échec scolaire. Des collégiens devaient mémoriser puis reproduire une figure complexe adaptée de la figure de Rey. Pour la moitié des élèves, cette tâche était présentée comme une évaluation de leurs aptitudes en géométrie. Pour l'autre moitié des élèves, il s'agissait d'une épreuve d'évaluation en dessin. Dans le contexte de la géométrie, la performance des élèves en échec scolaire était très inférieure à celle des élèves en réussite scolaire. Par contraste, dans le contexte des arts plastiques, la performance des élèves en échec scolaire était meilleure et ne différait plus de celle des élèves en situation de réussite. Ces résultats ont confirmé que les performances des élèves sont étroitement liées au contexte de présentation de la tâche ainsi qu'à la représentation que les élèves ont d'eux-mêmes dans telle ou telle discipline.

Le principal résultat des travaux sur les réputations individuelles ou collectives est que certaines différences de performances cognitives varient en fonction du contexte plus ou moins évaluatif de résolution des tâches. En ce sens, les différences observées ne semblent pas faire appel à des différences de réelles 
capacités intellectuelles. À partir de tels faits, les chercheurs ont construit des interprétations pour rendre compte des différences de performances entre les élèves de niveaux scolaires différents. Ces différences sont observées en fonction des modalités de présentation de la tâche. Les interprétations reposent sur des variables invoquées (liées aux sujets) dont on va chercher des indices de manifestation dans les comportements des élèves. Pour certains élèves, les contextes d'évaluation seraient coûteux en termes de ressources attentionnelles (Beilock \& Carr, 2005 ; Beilock, Kulp, Holt et al., 2004 ; Gimmig, Huguet, Caverni et al., 2006). Pour Monteil et ses collaborateurs (Brunot, Huguet \& Monteil, 2000 ; Monteil \& Huguet, 1999), l'origine de l'interférence est à rechercher dans la récupération des souvenirs personnels d'échec et des contenus émotionnels qui s'y rattachent.

En outre, les mécanismes sous-jacents à la mise en œuvre de la production orthographique ont été largement étudiés par la psychologie cognitive (Fayol, 1997 ; Fayol \& Jaffré, 2008). C'est l'analyse des erreurs orthographiques qui permet au moins partiellement d'identifier les processus intervenant dans la gestion des activités orthographiques. Ainsi, lorsque la charge en attention ou en mémoire est trop élevée pour permettre de gérer l'ensemble des contraintes (Fayol \& Jaffré, 1999), les sujets font appel à des modes de traitement généralement efficaces qui consistent soit à appliquer une procédure fréquente (accorder avec le nom le plus proche), soit à récupérer directement en mémoire une forme toute fléchie mais qui, dans les conditions de la recherche ou de la rédaction laborieuse, ne suffisent pas, un contrôle devant être exercé (Fayol, Hupet \& Largy, 1999). Notons toutefois que, jusqu'alors, seules les erreurs de type morphologique se sont avérées sensibles à l'augmentation de la charge. II ne s'ensuit pas que des erreurs d'orthographe dite lexicale ne puissent apparaître ; simplement, la mise en évidence de telles erreurs consécutivement à l'accroissement de la charge n'a pas encore été réalisée (Chanquoy, Tricot \& Sweller, 2007 ; Fayol \& Miret, 2005). Jusqu'alors, toutes les recherches manipulant la charge l'ont fait en recourant à des tâches secondaires mais, à notre connaissance, aucune n'a montré que différents contextes évaluatifs scolaires peuvent jouer le même rôle que ces tâches secondaires, à savoir augmenter (ou diminuer) le coût en attention ou en mémoire et, de ce fait, influer sur la fréquence et la nature des erreurs orthographiques produites par les élèves. L'analyse des erreurs commises lors de l'écriture d'un texte sous dictée apparaît comme un bon moyen d'étudier l'éventuel impact de différents contextes évaluatifs.
Aussi l'objectif de cette recherche sera-t-il d'évaluer l'effet de différentes situations scolaires évaluatives liées à l'activité de dictée. En classe, cet exercice est présenté de manière différente selon les objectifs des enseignants. Si les objectifs s'inscrivent dans la logique d'une évaluation sommative, l'enseignant choisira, le plus souvent, d'annoncer à ces élèves une dictée notée, comptant pour les résultats de fin de trimestre. En revanche, si les objectifs de l'enseignant sont liés à une évaluation formative, il présentera l'exercice de la dictée comme une étape dans le processus d'apprentissage et cet exercice ne sera pas noté. Dès lors, en référence aux travaux publiés dans le champ de la régulation sociale des performances (voir par exemple Monteil \& Huguet, 2002 ; Toczek \& Martinot, 2004 ; Dutrévis, Toczek \& Buchs, 2010), selon les objectifs pédagogiques de l'enseignant, cet exercice pourrait représenter une menace plus ou moins importante pour les élèves. Non notée, une dictée pourrait être peu menaçante pour les élèves. À l'inverse, notée et comptant pour le bilan trimestriel, cette même dictée pourrait représenter une condition plus menaçante, surtout pour les élèves faibles en orthographe. Par ailleurs, le texte correspondant à cette dictée pourrait être écrit sous dictée par les élèves dans un autre contexte que celui de l'orthographe ; ce contexte nous servirait alors de condition contrôle. Ainsi, si cette écriture de texte sous dictée prenait place dans une discipline à faible enjeu social, un tel contexte serait exempt de toute menace de réussite ou d'échec pour tous les élèves. Comme dans les études de Monteil et Huguet (Monteil \& Huguet, 1991 ; Huguet, Brunot \& Monteil, 2001), mais ici à travers une activité orthographique, deux contextes disciplinaires contrastés seront opérationnalisés : une activité mobilisant explicitement l'orthographe et une activité ayant trait aux arts plastiques. Ce contexte « arts plastiques » représente la condition contrôle - le contexte " sans évaluation »-, alors que le contexte « orthographe-dictée » représente le contexte disciplinaire relatif aux savoirs fondamentaux. Afin de nous situer dans le cadre de pratiques pédagogiques habituelles, nous opérationnalisons l'activité de dictée en ajoutant une consigne annonçant soit une absence de note, soit une note comptant pour la moyenne. Ainsi, trois situations (" sans évaluation et arts plastiques ", " dictée non notée et orthographe sans enjeu évaluatif " et « dictée notée et orthographe avec enjeu évaluatif ") ont été mises en place et caractérisent la première variable indépendante. De plus, l'histoire scolaire des élèves (c'est-à-dire leurs expériences de réussite et d'échec) étant identifiée par la littérature scientifique comme une variable déterminante dans la régulation psychosociale des performances (Monteil \& Huguet, 
2002), nous avons choisi de l'opérationnaliser par le niveau scolaire - fort versus faible - des élèves en orthographe. Cette variable est incluse dans notre protocole expérimental comme la seconde variable indépendante.

\section{HYPOTHÈSE}

Dans la continuité des travaux publiés jusqu'alors, nous posons une hypothèse d'interaction entre la situation scolaire des enfants (niveau fort versus faible) et les conditions de réalisation d'une dictée. Les recherches en psychologie sociale nous conduisent à prédire que l'enjeu évaluatif affecté à la production orthographique devrait influencer les performances des élèves en fonction de leur niveau scolaire initial. Ainsi, lorsque la tâche revêt un caractère menaçant pour les élèves de faible niveau scolaire (c'est-àdire « dictée notée » ou « contexte orthographe avec enjeu évaluatif »), l'écart de performances devrait être significatif entre les élèves de bon et de faible niveau scolaire, à l'avantage des premiers. De plus, nous attendons une accentuation de ces effets par rapport à la nature même des erreurs orthographiques des élèves. Nous pensons que les effets seront importants pour les erreurs de type morphologique. Néanmoins, l'écart de performance entre les deux groupes d'élèves forts et faibles en orthographe devrait être moindre dans le contexte sans pression évaluative (« orthographe sans enjeu évaluatif ") et inexistant dans la condition contrôle « arts plastiques ».

\section{MÉTHODE}

\section{Sujets}

Soixante-six élèves (vingt-cinq garçons et quarante et une filles) de $\mathrm{CM}_{2}{ }^{1}$, inscrits dans trois écoles de l'agglomération clermontoise, ont participé à cette expérience. En fonction de leur niveau initial en orthographe (élevé versus faible), les élèves sont répartis aléatoirement dans les trois contextes évaluatifs des performances.

\section{Procédure}

Chaque élève devait participer à trois séances de travail. L'objectif de la première séance était double : s'assurer que les trois classes avaient un niveau équivalent en orthographe et déterminer le niveau en orthographe de chacun des élèves. Au cours de cette séance, les élèves devaient réaliser deux tâches. La première - inspirée du test $\mathrm{ROC}$, repérage orthographique collectif, élaboré par Lecourvoisier, Boudinot, Davy-Aubertin et alii (2006) - consistait à identifier des erreurs d'orthographe à partir d'un texte et à les corriger. La seconde tâche (Fayol, Toczek, Labareyre et al., 2006) était présentée sous la forme d'un questionnaire à choix multiple et consistait à retrouver la forme orthographique correcte d'un mot inséré dans une phrase. Ces deux tâches sont présentées en annexe 1. La séance suivante était destinée à mettre en place un contexte de travail spécifique selon les conditions expérimentales : arts plastiques ou orthographe-dictée. Cette seconde séance ${ }^{2}$ avait pour seul objectif de rendre crédible le contexte de travail de la troisième séance, séance où se déroulait l'expérimentation proprement dite. Lors de cette troisième et dernière séance, les élèves ont écrit un texte sous dictée (voir l'annexe 2) dans trois contextes de travail scolaire qui caractérisent les trois conditions expérimentales testées dans cette étude. II s'agit d'un premier contexte de travail dont l'objet principal est une production en arts plastiques où les élèves écrivent de manière incidente un texte qui leur est dicté, d'un second contexte correspondant à une situation classique de dictée sans enjeu évaluatif (dictée non notée) et d'un troisième contexte caractérisé par une situation de dictée avec un enjeu évaluatif (dictée notée).

Tableau 1. Nombre moyen d'erreurs orthographiques selon la classe

\begin{tabular}{|c|c|c|c|c|c|}
\cline { 3 - 5 } \multicolumn{2}{c|}{} & \multicolumn{2}{c|}{ Test inspiré du test ROC } & \multicolumn{2}{c|}{ Test issu de Fayol et al. (2006) } \\
\hline Classe & Nombre d'élèves & $\begin{array}{c}\text { Nombre moyen } \\
\text { d'erreurs }\end{array}$ & Écart type & $\begin{array}{c}\text { Nombre moyen } \\
\text { d'erreurs }\end{array}$ & Écart type \\
\hline A & 23 & 5,5 & 2,2 & 24,9 & 5,8 \\
\hline B & 19 & 5,8 & 2,3 & 27,1 & 4,6 \\
\hline C & 24 & 5,5 & 2,6 & 27,7 & 4,8 \\
\hline
\end{tabular}




\section{RÉSULTATS}

Afin de nous assurer que les élèves des trois classes avaient un niveau équivalent en orthographe, nous avons comparé les performances des élèves aux deux épreuves permettant d'évaluer le niveau de base des élèves (voir le tableau 1). Pour chacun de ces scores, l'analyse statistique ne met en évidence aucune différence significative entre les trois classes $(F<1)$.

Ces épreuves nous ont permis de déterminer le niveau d'orthographe de chaque élève. Ainsi nous avons pu affecter à chaque situation expérimentale autant d'élèves d'un niveau élevé que d'élèves d'un faible niveau en orthographe. Nous avons catégorisé les élèves par rapport à la médiane. Une première analyse de variance (ou ANOVA ${ }^{3}$ ) $3 \times 2$, à savoir trois contextes (arts plastiques, dictée non notée, dictée notée) par deux niveaux en orthographe (élevé versus faible) a été réalisée sur le nombre moyen d'erreurs produites. Cette analyse de variance révèle un effet principal du niveau initial en orthographe $\left(F(1,65)=14,41 ; p<0,0001 ; \eta^{2}=0,19\right)$ : les élèves dont le niveau scolaire en orthographe est faible font plus d'erreurs orthographiques $(M=22,9)$ que les élèves d'un niveau élevé $(M=14,4)$, et ce, quel que soit le contexte scolaire (voir le tableau 2). Un effet principal du contexte $\left(F(2,65)=3,59 ; p<0,03 ; \eta^{2}=0,11\right)$ est également révélé par l'analyse de variance. Quel que soit leur niveau initial, les élèves produisent plus d'erreurs orthographiques dans la condition " arts plastiques " $(M=22,3)$ et moins d'erreurs dans la condition "dictée notée » $(M=15,1)$. Contrairement à l'hypothèse d'interaction posée dans cette étude, l'analyse de variance réalisée sur le nombre moyen d'erreurs produites révèle que le niveau des élèves en orthographe n'interagit pas avec le contexte de la tâche $(F<1)$. Contrairement à nos attentes, le contexte où l'enjeu évaluatif est le plus fort est associé aux meilleures performances des élèves, aussi bien pour les élèves d'un niveau élevé en orthographe que pour les élèves dont le niveau initial est plus faible.

Afin d'affiner cette première analyse, une seconde ANOVA $3 \times 2$, à savoir trois contextes (arts plastiques, dictée non notée, dictée notée) par deux niveaux en orthographe (élevé versus faible) a été réalisée pour différents types d'erreurs ${ }^{4}$. En effet, après avoir examiné l'effet de nos deux variables indépendantes sur le nombre total d'erreurs, il semble intéressant d'examiner l'effet de ces mêmes variables sur les différents types d'erreurs. Les contextes évaluatifs manipulés ici peuvent-ils jouer le même rôle que les tâches secondaires, à savoir augmenter ou diminuer le coût en attention ou en mémoire et, de ce fait, influer sur la fréquence et la nature des erreurs orthographiques produites par les élèves?

L'ensemble de ces traitements révèle le même pattern de résultat que celui obtenu lors de la première ANOVA : un effet du niveau initial des élèves en orthographe et une absence d'effet d'interaction entre les variables " niveau " et " contexte ". Plus précisément, un effet principal du niveau est mis en évidence $\left(F(1,65)=4,05 ; p<0,04 ; \eta^{2}=0,10\right)$ pour le nombre moyen d'erreurs phonogrammiques sans altération de la valeur phonique (comme pour " boté »/« beauté »). Les élèves de niveau élevé commettent moins d'erreurs de ce type $(M=2,8)$ par rapport aux élèves de niveau faible $(M=4,4)$. Un effet principal du niveau est également mis en évidence pour le nombre moyen d'erreurs de type phonogrammique avec altération de la valeur phonique, comme pour « chase »/« chasse » $\left(F(1,65)=9,01 ; p<0,004 ; \eta^{2}=0,14\right)$. Les bons élèves en orthographe produisent moins d'erreurs de ce

\section{Tableau 2. Nombre moyen d'erreurs orthographiques selon le niveau des élèves en orthographe (élevé versus faible) et selon le contexte}

\begin{tabular}{|c|c|c|c|c|c|c|c|}
\hline \multirow[b]{2}{*}{ Contexte } & \multicolumn{3}{|c|}{ Niveau élevé } & \multicolumn{3}{|c|}{ Niveau faible } & \multirow[b]{2}{*}{$\begin{array}{c}\text { Nombre moyen } \\
\text { d'erreurs selon } \\
\text { le contexte }\end{array}$} \\
\hline & $\begin{array}{l}\text { Nombre } \\
\text { d'élèves }\end{array}$ & $\begin{array}{c}\text { Nombre } \\
\text { moyen } \\
\text { d'erreurs }\end{array}$ & $\begin{array}{l}\text { Écart } \\
\text { type }\end{array}$ & $\begin{array}{l}\text { Nombre } \\
\text { d'élèves }\end{array}$ & $\begin{array}{c}\text { Nombre } \\
\text { moyen } \\
\text { d'erreurs }\end{array}$ & $\begin{array}{l}\text { Écart } \\
\text { type }\end{array}$ & \\
\hline Arts plastiques & 12 & 17,5 & 9,5 & 12 & 27,2 & 12,1 & 22,3 \\
\hline Dictée & 10 & 15,7 & 9,7 & 10 & 21,5 & 9,3 & 18,6 \\
\hline Dictée évaluée & 12 & 10,1 & 7,1 & 10 & 20,2 & 4,5 & 15,1 \\
\hline $\begin{array}{l}\text { Nombre moyen } \\
\text { d'erreurs selon } \\
\text { le niveau des élèves }\end{array}$ & & 14,4 & & & 22,9 & & \\
\hline
\end{tabular}


type $(M=2,4)$ en comparaison des élèves de niveau faible $(M=4,9)$. Et enfin, un effet principal du niveau initial en orthographe est noté pour les erreurs de type morphogrammique grammatical comme pour « les oiseau »/«les oiseaux " $(F(1,65)=18,08 ; p<0,0001$; $\eta^{2}=0,19$ ). Les élèves d'un niveau élevé en orthographe produisent moins d'erreurs de ce type $(M=5,3)$ en comparaison avec les élèves de niveau plus faible $(M=7,6)$. De plus, pour cet indicateur, un effet tendanciel du contexte est mis en évidence par l'analyse de variance $\left(F(1,65)=2,96 ; p<0,06 ; \eta^{2}=0,11\right.$ avec $M_{\text {Arts plastiques }}=6,9 ; M_{\text {Dictée }}=7,0 ; M_{\text {Dictée évaluée }}=5,6$ ). Notre hypothèse avançant une interaction entre ces deux variables, nous avons réalisé des analyses de contrastes. Des contrastes planifiés de type -1 (arts plastiques) et -1 (dictée) contre 2 (dictée évaluée) révèlent un seul effet significatif. L'écriture d'un texte dicté en vue d'être évalué conduit les bons élèves en orthographe à produire moins d'erreurs orthographiques de type morphogrammique grammatical que l'écriture d'un texte dicté sans l'enjeu d'une évaluation $\left(F(2,31)=4,29 ; p<0,05 ; \eta^{2}=0,10\right)$. Même s'il n'était pas attendu, ce résultat, fort intéressant, sera discuté.

\section{DISCUSSION}

La présente étude évalue les effets de trois contextes scolaires sur la mise en œuvre des performances orthographiques des élèves lors d'une activité d'écriture d'un texte dicté. En effet, même si certains travaux (par exemple Fayol, Hupet \& Largy, 1999 ; Largy, 2003 ; Largy, Cousin \& Dédeyan, 2005) s'intéressent à l'influence du contexte de la phrase, à l'effet du niveau d'expertise ou à l'effet de la nature de la tâche sur les performances orthographiques, aucune recherche ne s'était intéressée jusqu'alors à l'impact de différents contextes scolaires sur la mise en œuvre des performances orthographiques. Dès lors, en nous basant sur des travaux en psychologie sociale montrant que l'enjeu évaluatif affecté à une production intellectuelle influence les performances des élèves en fonction de leur niveau, nous prédisions un effet d'interaction entre les variables « niveau » et " contexte ". En d'autres termes, nous attendions que plus le contexte serait caractérisé par un enjeu évaluatif élevé et plus les performances des élèves ayant un faible niveau orthographique seraient basses. Sans surprise, nos résultats révèlent un effet du niveau initial des élèves sur l'ensemble des indicateurs caractérisant la mise en œuvre des performances orthographiques. En revanche, contrairement à notre hypothèse, aucun effet d'interaction n'est révélé ici. À l'inverse, le traitement des données recueillies ici infirme et même contredit notre hypothèse de départ. En effet, pour les deux groupes d'élèves (forts et faibles), le contexte sans pression évaluative "arts plastiques " se révèle le contexte le plus néfaste à la mise en œuvre des performances orthographiques des élèves, et ce pour le nombre total d'erreurs ainsi que pour les erreurs de type morphogrammique grammatical. Dès lors, ces résultats interpellent un certain nombre de travaux réalisés en psychologie sociale.

Notre recherche avait pour objectif d'évaluer les effets de contextes scolaires ancrés dans des pratiques pédagogiques ordinaires sur la mise en œuvre de performances orthographiques, en prenant en compte le niveau scolaire des élèves. Or notre principal résultat s'inscrit en contradiction avec les travaux publiés sur l'influence des contextes sur les performances, notamment les travaux d'Huguet, Brunot et Monteil (2001). Face à ces résultats antinomiques, une hypothèse explicative pourrait tenir au fait que dans cette recherche, la réplication du phénomène a été empêchée par la nature même de la tâche. Cette tâche est caractérisée par une activité scolaire - écriture d'un texte dicté - et est donc dépendante du niveau de base réel des élèves, contrairement aux études citées précédemment, où la tâche utilisée était une tâche de mémorisation indépendante de tout niveau scolaire préalable. Ces premiers résultats remettent en cause l'applicabilité de certaines recherches publiées dans le champ de la psychologie, recherches parfois trop éloignées des contextes scolaires. Mais avant d'aller aussi loin, nous pouvons concevoir une autre explication de ces résultats. Dans notre étude, la discipline qui sert de support à l'exercice est l'orthographe. Or cette discipline n'a vraisemblablement pas la même importance pour la réussite des élèves que le contexte de la géométrie, mobilisé dans les études réalisées auparavant. La géométrie représente en effet une discipline à fort enjeu social, alors que l'orthographe est classée comme la discipline la moins importante pour des élèves français (Dutrévis \& Toczek, 2007). Ainsi, non reliée à la réussite scolaire pour les élèves, l'orthographe, et plus particulièrement la dictée, ne favoriserait pas la focalisation sur les connaissances de soi, notamment sur le faible niveau orthographique. C'est pourquoi les élèves pourraient se focaliser plus facilement sur la tâche elle-même.

Dès lors, les résultats obtenus dans cette recherche soulignent le fait que l'enjeu évaluatif a des effets bénéfiques sur les performances orthographiques. L'évocation de cet enjeu semble agir sur la focalisation 
des ressources attentionnelles des élèves. Ainsi, focalisés sur la tâche, les élèves ont une meilleure gestion de l'orthographe, sans doute grâce à une révision orthographique effective. Ce résultat, s'il est confirmé par d'autres travaux complémentaires, pourrait avoir quelque utilité pour les pratiques enseignantes. Cette même hypothèse explicative semble convenir pour les élèves quel que soit leur niveau de base. Les recherches en psychologie cognitive rejoignent l'hypothèse explicative mobilisée par les psychologues sociaux. En effet, pour Just et Carpenter (1992) ou McCutchen (1996), un lot unique de ressources cognitives permet de traiter les informations langagières. Ainsi, lorsque les demandes des opérations en cours sont plus importantes que la quantité de ressources disponibles, alors certains traitements ou même le stockage temporaire sont dégradés ou freinés. Pour s'accommoder de cette mécanique, le lecteur ou le producteur de texte a intérêt à automatiser une partie des processus ou procédures afin de pouvoir gérer l'ensemble des demandes en ressources attentionnelles impliquées dans la tâche (Olive \& Piolat, 2005). Dès lors, dans cette étude, la théorie capacitaire pourrait expliquer la qualité de la production orthographique des élèves. D'ailleurs nos résultats mettent en évidence un effet tendanciel sur les erreurs de type morphologique, les erreurs les plus coûteuses au niveau attentionnel. C'est à nouveau lorsque l'enjeu évaluatif est élevé que la gestion des ressources attentionnelles est la meilleure.

La littérature sur les buts d'accomplissement (Dweck, 1986 ; Elliot \& Dweck, 1988 ; Nicholls, 1984) pourrait également expliquer ce résultat. En effet, le contexte évaluatif pourrait fixer aux élèves un but de réussite de type performance-approche tout à fait bénéfique, surtout pour les élèves dont le niveau en orthographe est élevé. Ces buts de performance-approche sont corrélés avec plus d'engagement et de persistance dans la tâche ou même avec de meilleures notes (Harackiewicz, Barron, Tauer et al., 2002 ; Darnon, Butera, Mugny et al., 2009). En fait, il semble qu'en orientant l'attention des élèves sur leurs résultats, ce type de but soit relativement favorable, mais qu'il n'encourage pas les élèves à effectuer des traitements profonds de la tâche (Midgley, Kaplan \& Middleton, 2001). Nous pouvons donc penser que ce type de but est favorable uniquement lorsque les apprentissages ont été automatisés par les élèves. Ici seuls les bons élèves ont de meilleures performances concernant les erreurs de type morphogrammique grammatical dans le contexte « dictée notée ».

D'une manière générale, tout se passe comme si l'enjeu évaluatif, habituellement un enjeu aux effets néfastes pour les performances des élèves en difficulté, se révélait le contexte le plus bénéfique pour tous. Cet enjeu évaluatif semble fixer des buts de performance à l'activité « dictée », lesquels permettent aux élèves de focaliser leur attention et de produire moins d'erreurs d'orthographe. La discipline orthographe étant une discipline peu importante pour les élèves, ces derniers ont, semble-t-il, besoin d'un enjeu évaluatif fort pour focaliser leur attention de manière efficace. En prolongement à cette recherche, une comparaison de ce même protocole au sein d'autres disciplines semblerait intéressante afin de comparer les effets de pratiques pédagogiques ordinaires de plusieurs disciplines scolaires aux enjeux contrastés.

Nous tenons à remercier Marylène Chalard, professeur des écoles, pour son aide précieuse lors du traitement des données.

Marie-Christine Toczek mctoczek@univ-bpclermont.fr Université Blaise-Pascal, Activité, connaissance, transmission, éducation

Michel Fayol michel.fayol@univ-bpclermont.fr Université Blaise-Pascal, laboratoire de psychologie sociale et cognitive

Marion Dutrévis marion.dutrevis@unige.ch Université de Genève (Suisse), faculté de psychologie et des sciences de l'éducation

\section{NOTES}

1 Nous avons choisi des classes de CM2 dont les enseignants proposaient régulièrement l'exercice de dictée, et ce avec des barèmes de notation et des fréquences évaluatives équivalentes.

2 Lors de cette séance, l'enseignant (en fait l'expérimentateur) se présentait aux élèves comme spécialiste soit des arts plastiques, soit de l'orthographe, et proposait une activité de type exercice (étude d'œuvres pour les arts plastiques et dictée pour l'orthographe) dans les domaines respectifs.
3 ANOVA est l'acronyme d'« analysis of variance ».

4 Les erreurs de type morphogrammique lexical, idéogrammique ou bien les erreurs concernant les homophones grammaticaux et les homophones lexicaux ont également été recensées mais leur nombre étant très faible, leur analyse statistique s'avère non pertinente dans cette étude. 


\section{BIBLIOGRAPHIE}

BEILOCK S. \& CARR T. (2005). "When high-powered people fail: Working memory and "choking under pressure" in math ". Psychological Science, vol. 16, n०2, p. 101-105.

BEILOCK S., KULP C., HOLT L. \& CARR T. (2004). “ More on the fragility of performance: Choking under pressure in mathematical problem solving ". Journal of Experimental Psychology: General, vol. 133, n० 4, p. 584-600.

BRUNOT S., HUGUET P. \& MONTEIL J.-M. (2000). "Performance feedback and self-focused attention in the classroom: When past and present interact $"$ Social Psychology of Education, vol. 3, no 4, p. 277293.

CABANEL P. (2002). La République du certificat d'études. Histoire et anthropologie d'un examen. Paris: Belin.

CHANQUOY L., TRICOT A. \& SWELLER J. (2007). La charge cognitive. Théories et applications. Paris : Armand Colin.

CHERVEL A. (2006). Histoire de l'enseignement du français $d u X V I^{e}$ au $x x^{e}$ siècle. Paris : Retz.

DARNON C., BUTERA F., MUGNY G., QUIAMZADE A. \& HULLEMAN C. (2009). " "To complex for me!" Why do performance-approach and performance-avoidance goals predict exam performance? " European Journal of Psychology of Education, vol. 24, no 4, p. 423-434.

DUTRÉVIS M. \& TOCZEK M.-C. (2007). «Perception des disciplines scolaires et genre des élèves : le cas de l'école primaire en France ". L'orientation scolaire et professionnelle, vol. 36, n³, p. 379-400.

DUTRÉVIS M., TOCZEK M.-C. \& BUCHS C. (2010). "Régulation sociale des apprentissages scolaires". In M. Crahay \& M. Dutrévis (dir.), Psychologie des apprentissages scolaires. Bruxelles : De Boeck, p. $85-110$

DWECK C. (1986). « Motivational processes affecting learning ". American Psychologist, vol. 41, n० 10, p. 1040-1048.

ELLIOT E. \& DWECK C. (1988). « Goals: An approach to motivation and achievement ". Journal of Personality and Social Psychology, vol. 54, no 1, p. 5-12.

FAYOL M. (1997). Des idées au texte. Paris : PUF.

FAYOL M. \& JAFFRÉ J.-P. (1999). « L'acquisition/ apprentissage de l'orthographe ". Revue française de pédagogie, no 126, p. 143-170.

FAYOL M. \& JAFFRÉ J.-P. (2008). Orthographier. Paris : PUF.

FAYOL M. \& MIRET A. (2005). «Écrire, orthographier et rédiger des textes ". Psychologie française, vol. 50, n० 3, p. 391-402.

FAYOL M., HUPET M. \& LARGY P. (1999). «The acquisition of subject-verb agreement in written French: From novice to expert's errors ». Reading and Writing, vol. 11, no 2 , p. 1-22.

FAYOL M., TOCZEK M.-C., LABAREYRE S. de \& CAILLAUD E. (2006). «Une brève épreuve d'évaluation des performances en morphologie flexionnelle écrite ". Rééducation orthophonique, n²25, p. 75-91.
GIMMIG D., HUGUET P., CAVERNI J.-P. \& CURY F. (2006). " Choking under pressure and working memory capacity: When performance pressure reduces fluid intelligence ». Psychonomic Bulletin \& Review, vol. 13, no 6, p. 1005-1010.

HARACKIEWICZ J., BARRON K., TAUER J. \& ELLIOT A. (2002). "Predicting success in college: A longitudinal study of achievement goals and ability measures as predictors of interest and performance from freshman year through graduation ". Journal of Educational Psychology, vol. 94, n० 3, p. 562-575.

HUGUET P., BRUNOT S. \& MONTEIL J.-M. (2001). "Geometry versus drawing: Changing the meaning of the task as a means to change performance ". Social Psychology of Education, vol. 4, n० 3-4, p. 219-234.

JUST M. \& CARPENTER P. (1992). « A capacity theory of comprehension: Individual differences in working memory ". Psychological Review, vol. 99, no 1, p. 122149.

LARGY P. (2003). “ Du contrôle de l'orthographe grammaticale. Première partie : du contrôle prégraphique ". Le langage et l'homme, vol. 38, n², p. 139-152.

LARGY P., COUSIN M.-P. \& DÉDEYAN A. (2005). « Produire et réviser la morphologie flexionnelle du nombre : de l'accès à une expertise ". Psychologie française, vol. $50, n^{\circ} 3$, p. 339-350.

LECOURVOISIER F., BOUDINOT A., DAVY-AUBERTIN C. \& WILLHELM C. (2006). ROC. Repérage orthographique collectif à l'usage des enseignants de fin de cycle 3 et de $6 \mathrm{e} / 5^{\mathrm{e}}$ de collège. Document de travail. Grenoble : IUFM de Grenoble, laboratoire Cogni-sciences. En ligne: http://www.cognisciences.com/article.php3?id_ article=42 (consulté le 14 février 2012).

MANESSE D. (2007). "La dictée, résistance et avatars d'un exercice scolaire ». In H. Peyronie \& A. Vergnioux (dir.), Éducation et longue durée. Caen : Presses universitaires de Caen.

McCUTCHEN D. (1996). "A capacity theory of writing: Working memory in composition ". Educational Psychology Review, vol. 8, n³, p. 299-325.

MIDGLEY C., KAPLAN A. \& MIDDLETON M. (2001). "Performance-approach goals: Good for what, for whom, under what circumstances, and at what cost? " Journal of Educational Psychology, vol. 93, no 1, p. 7786.

MONTEIL J.-M. \& HUGUET P. (1991). «Insertion sociale, catégorisation sociale et activités cognitives ". Psychologie française, vol. 36, no 1, p. 35-46.

MONTEIL J.-M. \& HUGUET P. (1999). Social context and cognitive performance: Towards a social psychology of cognition. Hove : Psychology Press.

MONTEIL J.-M. \& HUGUET P. (2002). Réussir ou échouer à l'école : une question de contexte ? Grenoble : Presses universitaires de Grenoble.

NICHOLLS J. (1984). « Achievement motivation: Conceptions of ability, subjective experience, task choice, and performance ". Psychological Review, vol. 91, n॰3, p. 328-346. 
OLIVE T. \& PIOLAT A. (2005). « Le rôle de la mémoire de travail dans la production écrite de textes ». Psychologie française, vol. 50, no 3, p. 373-390.

SCHMADER T. \& JOHNS M. (2003). " Converging evidence that stereotype threat reduces working memory capacity ». Journal of Personality and Social Psychology, vol. 85, n॰3, p. 440-452.
STEELE C. (1997). " A threat in the air: How stereotypes shape the intellectual identities and performance of women and African-Americans ". American Psychologist, vol. 52, n० 6, p. 509-516.

TOCZEK M.-C. \& MARTINOT D. (2004). Le défi éducatif. Des situations pour réussir. Paris : Armand Colin.

\section{ANNEXE 1. DESCRIPTIF DE LA PREMIÈRE SÉANCE DE TRAVAIL}

\section{Annexe 1a. Première épreuve de la séance 1}

Cette épreuve - inspirée du test ROC élaboré par Lecourvoisier, Boudinot, Davy-Aubertin et Willhelm (2006) consiste à identifier des erreurs d'orthographe à partir d'un texte et à les corriger.

La personne qui a tapé le texte a commis des erreurs d'orthographe. Elle a besoin de ton aide car elle n'arrive pas à les trouver. Peux-tu les souligner et les corriger juste en dessous ?

Exemple :

La mézon est jolie.

maison

Un jeune garson, passionné par la faune, se promené au zoo pour observait désanimaux sauvages. Le plus imposant d'entre eux, l'éléfant, lui parut bien domestiqué derrière sa grille ; les tigres et les lions ressemblai plus à de gros chats curieu qu'à de terribles prédateur. Toutacoup, l'enfant resta ébahi devant le dernier animal non domestiqué du parc. S'était un magnifique pijon perché avec orgueil sur un cayou. Lorsqu'il voulut le photographié, effrayé, l'oiseau sens vola retrouver sa liberté.

\section{Annexe 1b. Deuxième épreuve de la séance 1}

Cette épreuve consiste à retrouver la forme orthographique correcte d'un mot inséré dans une phrase (Fayol, Toczek, Labareyre et al., 2006).

$$
\text { cries }
$$

1. Les enfants crient au secours car ils sont perdus dans la forêt.

crie

amie

2. Paul a une ami.

amit

calcule

3. Les fillettes calcules combien elles ont dépensé.

calculent 
poules

4. Dans la basse-cour, les poule picorent du blé.

poulent

5. C'est la fête : les enfants chantent.

chante
chantent.
chantes

court

6. Le garçon cour jusqu'à la ligne d'arrivée.

cours

vide

7. Les vendeurs vides les casiers.

vident

téléphones

8. Les téléphonent sonnent.

téléphone

pointuent

9. Les crayons pointu sont rangés dans la trousse. pointus

10. Les enfants sont en récréation : ils jouent au ballon. joues

\section{portent}

11. Les portes de la maison sont vertes. porte

bavarde

12. Pendant la journée, les coiffeuses bavardes et travaillent.

bavardent

\section{chantes}

13. C'est Noël. Les enfants sont heureux : ils chante des airs joyeux.

chantent

$$
\text { timbres }
$$

14. J'ai oublié de coller les timbrent sur l'enveloppe.

timbre

portes

15. Elles portent ces objets avec précaution. porte

joues

16. Les petites filles s'amusent: elles joue à la poupée.

jouent

plantes

17. Les plante du fleuriste sont très belles. plantent

timbrent

18. Avant d'aller à la poste, les enfants timbres leurs lettres.

timbre 
calculent

19. Les calculs de cet exercice sont difficiles.

calcul

table

20. Les élèves apprennent les tablent de multiplication.

tables

remplacerai

21. Quand vous serez fatigués, je vous remplacerez. remplacerais

22. Les chiens fuient : il les appelles

appelles
appelle.

appellent

portent

23. Ces objets, il les portes avec précaution.

porte

vogue

24. Sur la mer voguent les voiliers.

vogues

relie

25. Avec son crayon elle relit les dessins semblables.

relis

glisse

26. Sur la patinoire glisses les danseurs.

glissent

vident

27. Le vendeur nettoie les casiers vide.

vides

28. II est 7 heures : le réveille sonne

réveil

Parlez

29. Parler plus fort! Je ne vous entends pas.

Parlé

Dépêcher

30. Dépêchez-vous! Le car va partir.

Dépêché

installer

31. Ils ont lentement installés les échafaudages. installé

téléphoner

32. II voulait téléphonait à sa sœur.

téléphoné

tomber

33. Il est tombé de sa chaise.

tombait 
34. Pendant les vacances, ils ont pu avancé dans leur travail.

avancer

reviendrai

35. C'est promis, je reviendrais vous voir demain.

reviendrez

36. Si vous me l'aviez demandé, je serai rentré plus tôt.

serais

bavardent

37. Les coiffeuses bavarde travaillent tout l'après-midi.

bavardes

\section{adressé}

38. La lettre qu'il leur a adressée a été perdue.

adressés

aboyer

39. Les chiens ont aboyés parce qu'ils ont vu un chat.

aboyé

emporté

40. Les voleurs ont emportés le coffre-fort.

emporter

\section{ANNEXE 2. OUTILS UTILISÉS LORS DES SÉANCES 2 ET 3}

\section{Annexe 2a. Texte utilisé au cours de la séance 2}

Un corbeau étrange nommé Léon, vit dans un vieux chêne. Habituellement, notre corbeau surveille ses proies du haut des plus hautes branches de son arbre. Il les repère rapidement. Aujourd'hui, perché sur l'antenne d'un bâtiment, Léon tient dans son bec une souris blessée. L'oiseau a des griffes pointues. II les referme sur sa proie.

\section{Annexe $2 b$. Texte dicté au cours de la séance 3 , dans les trois contextes étudiés}

Je vais vous raconter l'histoire d'un gentil petit garçon qui s'appelle Jo. II habite chez son oncle, un vieux monsieur qui habite dans un bourg. Cet enfant possède un don extraordinaire. En effet, grâce à ses yeux verts, il voit beaucoup plus loin et précisément que tout le monde! Dans ses pupilles se trouvent des jumelles intégrées, microscopiques et invisibles... 